\title{
SIMULAÇÃO DA SOLIDIFICAÇÃO DE UMA LIGA DE ALUMÍNIO-SILICIO EM UM PROÇESSO DE CINTO ÚNICO
}

\author{
Jose Adilson de Castro ${ }^{1}$ \\ Alexandre Furtado Ferreira ${ }^{2}$ \\ Alexandre José da Silva ${ }^{3}$
}

\begin{abstract}
Resumo: O presente trabalho tem como objetivo prever o processo de solidificação de uma liga de alumínio-silício através da simulação computacional. O modelo proposto inclui cinética de solidificação e os fenômenos relacionados ao transporte de energia, massa e difusão de soluto nas fases sólida e líquida. O modelo apresentado neste trabalho consiste na solução simultânea de equações de transporte de energia e conservação de espécies químicas nas fases sólida e líquida. As equações diferenciais parciais que modelam os fenômenos envolvidos são resolvidas através da técnica de volumes finitos. Propriedades de relevância para o modelamento foram coletadas da literatura para a liga considerada. Dentre os diversos parâmetros estudados através da simulação computacional da evolução da solidificação da liga depositada no substrato, escolheu-se devido à sua relevância, a influência da resistência de contato à transferência de calor do metal para o molde, a velocidade de lingotamento e a espessura da tira lingotada. Dentre os resultados mais relevantes estão a previsão da evolução temporal dos campos de temperatura e distribuição de soluto nas fases sólida e liquida, bem como a influência das diversas variáveis de processo. Os valores calculados para o campo de temperaturas e demais variáveis estão de acordo com valores medidos apresentados na literatura.
\end{abstract}

Palavras-chave: Lingotamento de tiras, simulação computacional, solidificação.

Abstract: This work aims to predict the solidification phenomenon of an alloy of aluminumsilicon using the computational simulation technique. The model proposed includes the solidification kinetics as well as the phenomena related to the energy and chemical species transport into the liquid and solid phases taking into account the solute redistribution phenomenon. The partial differential equations modeling the involved phenomena are solved based on the finite volume technique and the thermophysical properties are modeled by correlations reported in the literature. Among the simulation parameters, the thermal resistance between metal and mould was paid special attention due to its influence into the temperature distribution of the metal layer as well as the heat flux. In addition, the influence of the casting velocity and layer thickness of the solidified product were investigated. Among the important results the temperature and solute distribution fields at the solid and liquid phases are presented. The calculated results for the temperature and solute distribution fields are in good agreement with those measured formerly reported in the literature.

Key words: strip casting, numerical simulation and solidification

1,2,3 EEIMVR-UFF - E-mail: ${ }^{1}$ adilson@metal.eeimvr.uff.br, ${ }^{2}$ furtado@metal.eeimvr.uff.br, 3ajs@metal.eeimvr.uff.br 


\section{1 - Introdução}

Os processos de lingotamento de tiras finas permitem a produção de tiras diretamente a partir do metal líquido, eliminando ou simplificando significativamente as etapas de reaquecimento e laminação, com consequência da redução nos custos operacionais e de investimento no caso de novas plantas. Os diversos programas de pesquisa sobre o lingotamento contínuo de tiras demonstraram o grande interesse comercial nos possíveis benefícios desta tecnologia. Nos processos de cinto único, os principais processos que ocorrem são a solidificação, extração de calor pelo molde e vizinhanças, segregação de soluto e transformações de fase no estado sólido, dependendo da liga solidificada. Viskanta et alli $^{\text {ref0 }}$ apresentam uma revisão detalhada dos fenômenos de solidificação e propuseram a solução analítica para um problema unidimensional(problema de Stefan) que é usualmente uma primeira aproximação para $o$ tratamento do problema de solidificação em um cinto único. Guthrie e Tavares ${ }^{3)}$ modelaram o processo baseando-se em equações de transporte com a fração transformada na "mushy zone" data em função da temperatura, adicionando a parcela do calor latente na equação de transporte.

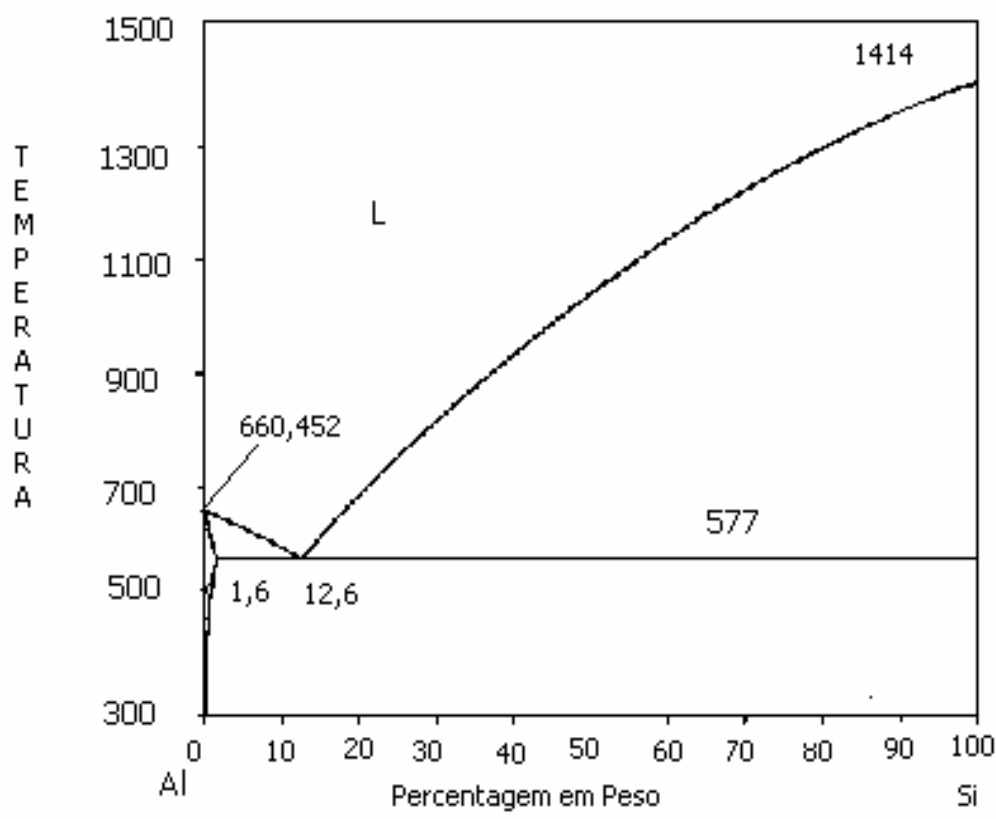

Figura 1 - Diagrama de fases $\mathrm{Al}-\mathrm{Si}^{\text {7) }}$

Portanto, diversos trabalhos visando o entendimento da fenomenologia e variáveis dos processos de cinto único tem sido desenvolvidos. Entre os mais recentes pode-se citar aqueles desenvolvidos no programa de pós graduação em Engenharia Metalúrgica em Volta Redonda.

Netto. P. G. Q. ${ }^{8)}$ em sua tese de doutoramento desenvolveu modelamento físico e matemático do processo de cinto único, estudando o escoamento, transferência de calor e massa em um modelo bidimensional. Neste estudo foram realizados experimentos visando estudar a influência da espessura da tira do coeficiente de troca de calor no contato metal/molde e da velocidade de lingotamento, concluindo que a espessura 
fina e alto superaquecimento aumenta o fluxo de calor interfacial.

Júnior J. M. F. ${ }^{8)}$ em sua tese de mestrado desenvolveu um trabalho que visou a construção de um simulador de lingotamento contínuo de tiras. Com a finalização deste trabalho chegou-se as seguintes conclusões: a) Um melhor entendimento do processo de deposição de metais em escala de laboratório, em máquina de cinto único, poderia ajudar na melhor compreensão do processo real de deposição de metais em substratos móveis, neste sentido, pode-se ressaltar a importância do trabalho desenvolvido por Júnior J. M. F. ${ }^{8)}$, uma vez que a continuidade deste projeto pode ser posteriormente implementada por Silva A. T. ${ }^{10)}$.

Silva A. T. ${ }^{10)}$ estudou a influência da forma de deposição de metal na qualidade de tiras produzidas em máquina de lingotamento de cinto único. Neste Trabalho Silva A. T. chegou às seguintes conclusões: a) A deposição do metal em ângulo de $45^{\circ}$ permite a produção de tiras com uma qualidade superficial superior aquelas lingotadas com um distribuidor reto, como consequência de um melhor contato metal/substrato; b) A deposição em ângulo de $45^{\circ}$ também resulta em taxas de resfriamento de metal superiores aquela ocorridas no caso do distribuidor reto. Essa conclusão tem como base os valores mais altos de fluxo de calor interfacial ocorridos na deposição a $45^{\circ}$. Os valores dos picos de fluxo para o caso do distribuidor de base inclinada foram cerca de $30 \%$ maiores que para o caso do distribuidor reto propiciando o surgimento de uma microestrutura mais refinada; c) Conclui-se também que as diferentes condições de alimentação de metal sobre o substrato móvel não divergem significativamente quanto a uniformidade dimensional e nem quanto à ocorrência de segregação nas tiras produzidas nessas condições.

Apesar de não serem tão populares quanto os processos de duplo-rolo, os processos que empregam um único cinto móvel apresentam vantagens bastante importantes. Estas vantagens estão associadas à produtividade e qualidade das tiras, produzidas em um ambiente livre de deformação, portanto o desenvolvimento destes processos é de grande interesse para a indústria siderúrgica moderna.

O objetivo do presente trabalho foi, então, adequar um modelo matemático para lidar com problemas de solidificação de uma liga Al-Si, obtida pelo processo de cinto único. Em particular, a adequação deste modelo permitirá a análise de transferência de calor visando o perfil térmico do sistema analisado, isto é, $\mathrm{T}=\mathrm{T}(\mathrm{x}, \mathrm{y}, \mathrm{z}, \mathrm{t})$, fração de produto solidificado ao longo do tempo, e ainda a influência da velocidade de lingotamento e da espessura da tira produzida.

O trabalho, aqui apresentado, representa uma continuidade na pesquisa e detalhamento de um modelo matemático que possa prever de maneira acurada os processos reais de lingotamento de tiras, com posterior análise de parâmetros tecnológicos de interesse para $\mathrm{o}$ aprimoramento do processo industrial bem como um melhor entendimento dos fenômenos que governam o mesmo. Neste sentido, este trabalho contribui para o refinamento do modelamento anteriormente proposto por outros pesquisadores, introduzindo um modelo bifásico com troca de calor e segregação de soluto em um domínio tridimensional. Adicionalmente o modelamento aqui apresentado engloba não somente os fenômenos que ocorre na tira a ser solidificada, mas também no molde, onde a resistência térmica de contato é um parâmetro importante, conforme demonstrado por outros pesquisadores $^{2,3,4,5,6)}$.

\section{O Modelamento Matemático}

O modelamento apresentado neste trabalho consiste na solução simultânea de 
equações de transporte de energia e conservação de espécies químicas nas fases sólida e líquida ${ }^{7-12)}$. Neste trabalho o modelo matemático foi adequado à solidificação de uma liga Al-Si. A figura 1 mostra o diagrama de fases desta liga, a tabela 1 mostra as propriedades térmicas dos materiais coletados da literatura e utilizados no desenvolvimento deste trabalho.

Embora a liga utilizada apresente traços de diversos elementos de liga, foi considerado apenas as espécies de alumínio e silício. Entretanto, as propriedades relevantes para o modelamento foram coletadas da literatura para a liga em questão. Foi dado especial atenção ao transporte das espécies químicas alumínio e silício, já que os mesmos representam quase a totalidade da composição.

Tabela 1 - Propriedades térmicas.

\begin{tabular}{|c|c|c|c|}
\hline MATERIAL & $\begin{array}{c}\mathrm{k} \\
(\mathrm{W} / \mathrm{m} . \mathrm{K})\end{array}$ & $\begin{array}{c}\text { Cp } \\
(\mathrm{J} / \mathrm{mol} . \mathrm{K})\end{array}$ & $\begin{array}{c}\rho \\
\left(\mathrm{Kg} / \mathrm{m}^{3}\right)\end{array}$ \\
\hline Molde & $64,1-0,0427 \mathrm{~T}$ & $4,13+0,00638 \mathrm{~T}$ & 7200 \\
\hline $\mathrm{Al}-8.4 \% \mathrm{Si}$ & $64(1), 161(\mathrm{~s})$ & $\begin{array}{c}7,0\left(\mathrm{Al}_{1}\right), 4,8+0,00322 \mathrm{~T}\left(\mathrm{Al}_{\mathrm{s}}\right), \\
5,74+0,000617 \mathrm{~T}\left(\mathrm{Si}_{1}, \mathrm{Si}_{\mathrm{s}}\right)\end{array}$ & 2680 \\
& & & \\
\hline
\end{tabular}

O modelo proposto consiste de um modelo bi-fásico tridimensional transiente $^{7,8)}$ onde são resolvidas simultaneamente 7 equações diferenciais parciais com apropriadas condições de contorno e iniciais.

Entalpia da fase líquida:

$$
\begin{aligned}
& \frac{\partial\left(\varepsilon_{1} \rho_{1} \mathrm{~h}_{1}\right)}{\partial \mathrm{t}}+\operatorname{div}\left(\varepsilon_{1} \rho_{1} \overrightarrow{\mathrm{U}}_{1} \mathrm{~h}_{1}\right) \\
& =\operatorname{div}\left(\frac{\mathrm{k}_{1}}{\mathrm{Cp}_{1}} \operatorname{grad}\left(\mathrm{h}_{1}\right)\right)+\mathrm{S}_{\mathrm{hl}}
\end{aligned}
$$

Entalpia da fase sólida:

$$
\begin{aligned}
& \frac{\partial\left(\varepsilon_{\mathrm{s}} \rho_{\mathrm{s}} \mathrm{h}_{\mathrm{s}}\right)}{\partial \mathrm{t}}+\operatorname{div}\left(\varepsilon_{\mathrm{s}} \rho_{\mathrm{s}} \overrightarrow{\mathrm{Us} \mathrm{h}}_{\mathrm{s}}\right) \\
& =\operatorname{div}\left(\frac{\mathrm{k}_{\mathrm{s}}}{\mathrm{Cp}_{\mathrm{s}}} \operatorname{grad}\left(\mathrm{h}_{\mathrm{s}}\right)\right)+\mathrm{S}_{\mathrm{h}_{\mathrm{s}}}
\end{aligned}
$$

Fração mássica do alumínio na fase líquida:

$$
\begin{aligned}
& \frac{\partial\left(\varepsilon_{1} \rho_{1} \omega_{\mathrm{Al}-1}\right)}{\partial \mathrm{t}}+\operatorname{div}\left(\varepsilon_{1} \rho_{1} \overrightarrow{\mathrm{U}}_{1} \omega_{\mathrm{Al}-1}\right) \\
& =\operatorname{div}\left(\rho_{1} \mathrm{D}_{\mathrm{Al}-1}^{\mathrm{Al}-1} \operatorname{grad}\left(\omega_{\mathrm{Al}-1}\right)\right)+\mathrm{S}_{\mathrm{Al}-1}
\end{aligned}
$$

Fração mássica de silício na fase líquida:

$$
\begin{aligned}
& \frac{\partial\left(\varepsilon_{1} \rho_{1} \omega_{\mathrm{Si}-1}\right)}{\partial \mathrm{t}}+\operatorname{div}\left(\varepsilon_{1} \rho_{1} \overrightarrow{\mathrm{U}}_{1} \omega_{\mathrm{Si}-1}\right) \\
& =\operatorname{div}\left(\rho_{1} \mathrm{D}_{\mathrm{Si}-1}^{\mathrm{Al}-1} \operatorname{grad}\left(\omega_{\mathrm{Si}-1}\right)\right)+\mathrm{S}_{\mathrm{Si}-1}
\end{aligned}
$$


Fração mássica do alumínio na fase sólida:

$$
\begin{aligned}
& \frac{\partial\left(\varepsilon_{\mathrm{s}} \rho_{\mathrm{s}} \omega_{\mathrm{Al}-\mathrm{s}}\right)}{\partial \mathrm{t}}+\operatorname{div}\left(\varepsilon_{\mathrm{s}} \rho_{\mathrm{s}} \overrightarrow{\mathrm{U}}_{\mathrm{s}} \omega_{\mathrm{Al}-\mathrm{s}}\right) \\
& =\operatorname{div}\left(\rho_{\mathrm{s}} \mathrm{D}_{\mathrm{Al}-\mathrm{s}}^{\mathrm{Al}-\mathrm{s}} \operatorname{grad}\left(\omega_{\mathrm{Al}-\mathrm{s}}\right)\right)+\mathrm{S}_{\mathrm{Al}-\mathrm{s}}
\end{aligned}
$$

Fração mássica do silício na fase sólida:

$$
\begin{aligned}
& \frac{\partial\left(\varepsilon_{\mathrm{s}} \rho_{\mathrm{s}} \omega_{\mathrm{Si}-\mathrm{s}}\right)}{\partial \mathrm{t}}+\operatorname{div}\left(\varepsilon_{\mathrm{s}} \rho_{\mathrm{s}} \vec{U}_{\mathrm{s}} \omega_{\mathrm{Si}-\mathrm{s}}\right) \\
& =\operatorname{div}\left(\rho_{\mathrm{s}} \mathrm{D}_{\mathrm{Si}-\mathrm{s}}^{\mathrm{Si}-\mathrm{grad}}\left(\omega_{\mathrm{Si}-\mathrm{s}}\right)\right)+\mathrm{S}_{\mathrm{Si}-\mathrm{s}}
\end{aligned}
$$

Fração de sólido:

$$
\begin{aligned}
& \frac{\partial\left(\rho_{\mathrm{s}} \varepsilon_{\mathrm{s}}\right)}{\partial \mathrm{t}}+\operatorname{div}\left(\rho_{\mathrm{s}} \overrightarrow{\mathrm{U}}_{\mathrm{s}} \varepsilon_{\mathrm{s}}\right) \\
& =\mathrm{S}_{\mathrm{Si}-\mathrm{s}}+\mathrm{S}_{\mathrm{Al}-\mathrm{s}}
\end{aligned}
$$

E adicionalmente a determinação da fração do líquido é determinada por:

$$
\varepsilon_{S}+\varepsilon_{l}=1
$$

Estas 7 equações diferenciais parciais são resolvidas simultaneamente através da técnica de volumes finitos ${ }^{9-10}$ ) onde o domínio é subdivido em volumes elementares. Adicionalmente, condições iniciais e de contorno devem ser especificadas.

\section{Condições Iniciais:}

Neste modelamento considera-se que o líquido é vazado no molde a uma temperatura em torno de $20^{\circ} \mathrm{C}$ acima da temperatura liquidus da liga considerada ( veja diagrama de equilíbrio, figura 1), portanto a todos os volumes elementares que corresponde ao domínio da tira é atribuída o seguinte campo de temperatura inicial $T(x, y, z, 0)=T_{l}+T_{s h}$ onde a temperatura $T_{1}$ é dada pelo diagrama de equilíbrio (figura 1) como função da composição da liga e $\mathrm{T}_{\mathrm{sh}}$ é a temperatura de superaquecimento. No restante do domínio ENGEVISTA, v. 6, n. 1, p. 35-46, abr. 2004 (molde), a temperatura inicial foi a ambiente $\left(25{ }^{\circ} \mathrm{C}\right)$. A composição inicial é dada pela composição da liga a ser solidificada. Neste trabalho adotou-se a seguinte composição inicial, que foi o valor atribuído inicialmente em todo o domínio da tira, enquanto na região do molde assumiu-se a composição do molde(aço):

$$
\begin{aligned}
& A l_{\text {inicial }}=88,5 \% \\
& S i_{\text {inicial }}=11,5 \%
\end{aligned}
$$

e a fração de sólido é dado por $\varepsilon_{\mathrm{S}}=0, \mathrm{t}=0$.

\section{Condições de Contorno:}

Para as interfaces em contato com o ar foi utilizado simultaneamente a convecção e radiação dada por:

$$
\begin{aligned}
& q_{\text {conv }}=h A\left(T_{\infty}-T_{W}\right) \\
& q=5,68 \times 10^{-8} \varphi A\left(T_{\infty}^{4}-T_{W}^{4}\right)
\end{aligned}
$$

$\mathrm{Na}$ interface metal-molde, Silva et $\mathrm{al}^{6)}$ realizou experimentos, onde através da medida de temperaturas associado à solução do problema inverso de troca de calor determinou o fluxo de calor através da interface. Neste modelo considera-se que na interface ocorre a troca de calor segundo o modelo Newtoniano.

$$
q_{\text {Newton }}=h_{\text {aparente }} A\left(T_{l}-T_{w}\right)
$$

O método de volumes finitos ${ }^{9-10)}$ foi utilizado para obter as equações discretizadas onde as equações diferenciais são transformadas em um conjunto de equações algébricas acopladas. A discretização temporal é baseada no método implicito ( Fully Implicit Method ) recomendado por Patankar ${ }^{10)}$ e Mellaen ${ }^{9)} \mathrm{O}$ sistema obtido é resolvido através do método iterativo "line by line" em conjunção com um sistema tri-diagonal. A malha de cálculo utilizada nesta simulação foi refinada na região da tira, pois 
representa a região onde os gradientes de temperatura são mais acentuados ao longo da simulação. $\mathrm{O}$ número total de volumes considerados na simulação foi de 9000 , sendo 6000 na região da tira e 3000 na região do molde. Para realização das simulações foi usado uma estação de trabalho ALPHA 600. O código simulador foi implementado em FORTRAN 90, sendo divido em três módulos básicos: Preprocessamento onde todos os dados de entrada e inicializações de todas as variáveis são realizados, a construção de uma malha estruturada (BFC-body Fitted Coordinate), $\mathrm{O}$ processamento onde estão as rotinas relacionadas ao solver e um módulo de Pos-Processamento, onde as saídas gráficas e geração de arquivos de dados são realizados. Como critério de convergência adotou-se um erro máximo da orden de $1 \times 10^{-03}$ para todos os valores do campo de temperatura.

Parte importante do procedimento de cálculo consiste na determinação do termo fonte de cada equação. Os termos fonte de energia e conservação de espécies químicas podem ser determinados quando o modelo de solidificação é definido. Existem vários modelos de solidificação propostos na literatura, entretanto todos assumem uma temperatura final de solidificação conhecida e portanto admitem que a liga ao atingir a temperatura liquidus instantaneamente é solidificada ${ }^{2,3}$. Neste trabalho um modelo de cinética de solidificação é proposto como segue.

\section{1 - Modelo de Solidificação}

equação:

Neste trabalho propõe-se que a taxa de solidificação é determinada através da seguinte A entalpia da fase na temperatura liquidus é dada por:

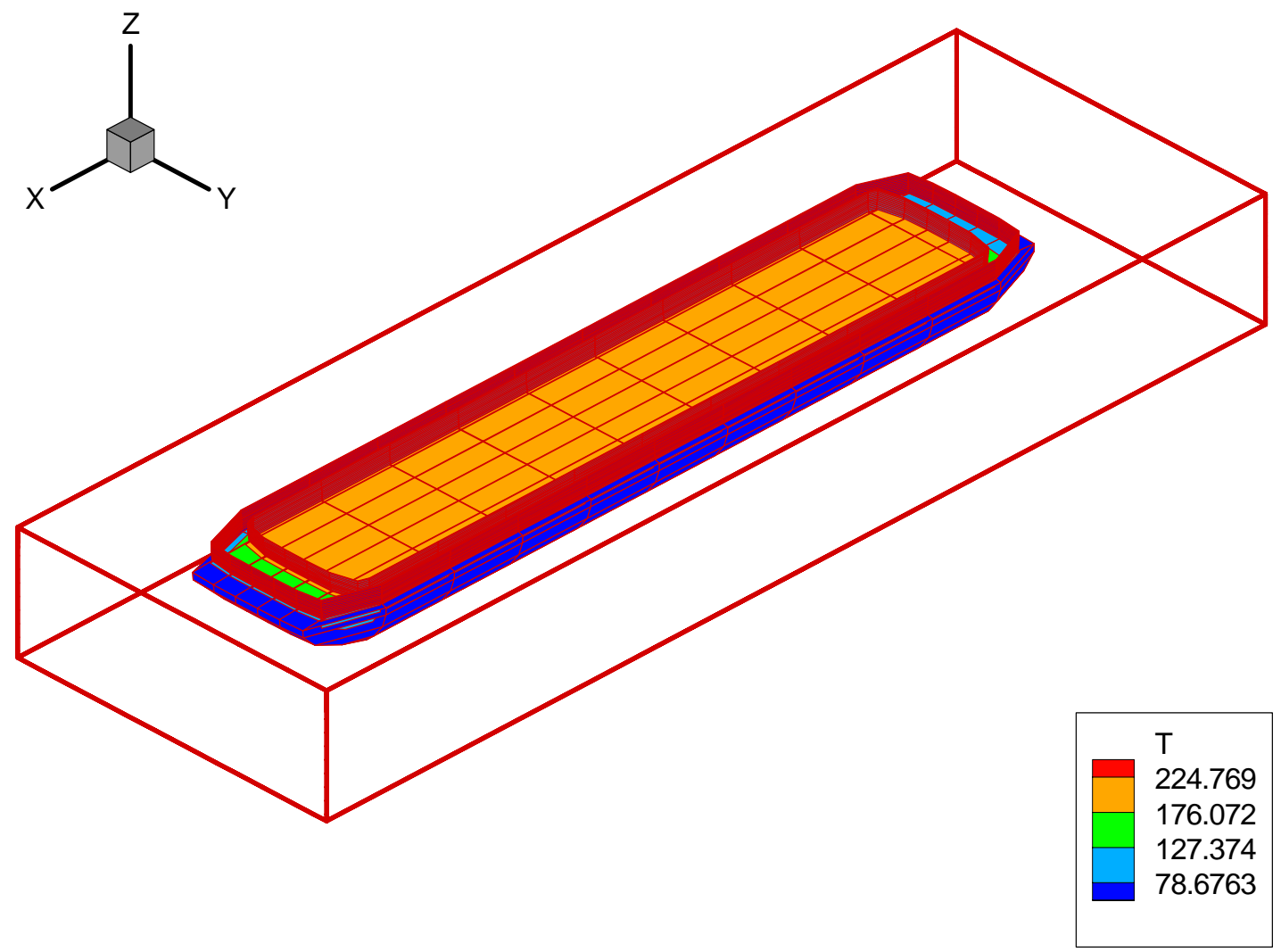

EN Figura 2. Simulação do sistema metal/molde tridimensional(região em branco representa gradiente de temperatura nulo). 


$$
\begin{aligned}
& H_{\text {Liquidus }}=a\left(T_{l}-298\right)+0,5 b\left(T_{L}^{2}-298^{2}\right) \\
& -c\left(\frac{1}{T_{L}}-\frac{1}{298}\right)
\end{aligned}
$$

Quando a temperatura decai abaixo da temperatura liquidus a entalpia seria:

$$
\begin{aligned}
& H_{T}=a(T-298)+0,5 b\left(T^{2}-298^{2}\right) \\
& -c\left(\frac{1}{T}-\frac{1}{298}\right)
\end{aligned}
$$

E a taxa de solidificação é dada pela equação abaixo, onde assume-se uma cinética de solidificação proporcional ao

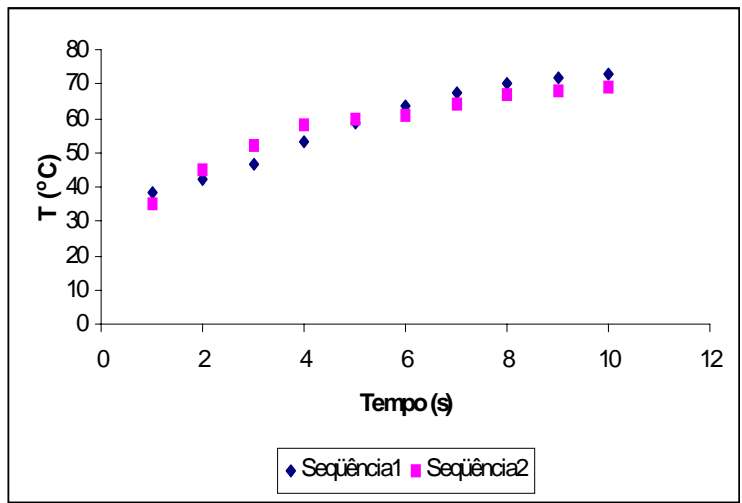

Figura 3a - Valores medidos versus valores previstos, com a resistência térmica de contato.

Os termos fontes para as equações de transporte de alumínio e silício no sólido são obtidos pelas equações (14) e (15), respectivamente.

$$
\begin{aligned}
& R_{S i}=R_{S} C_{S} \\
& R_{A l}=R_{S}\left(1-C_{S}\right)
\end{aligned}
$$

onde $\mathrm{C}_{\mathrm{s}}$ é admitido como sendo a fração mássica de saturação do silício no sólido, que varia com a temperatura conforme o diagrama de fases da figura 1.

ENGEVISTA, v. 6, n. 1, p. 35-46, abr. 2004 super-resfriamento, fator importante é a taxa de extração de calor local que depende da difusividade térmica do material e dos efeitos de conveção da fase líquida, entretanto a equação de conservação de energia é resolvida acoplada às equações de cinética da solidificação e transferência de soluto, o que acopla os diversos fenômenos, a solução iterativa das equações acima permite, então a obtenção da solução aproximada dos campos de temperatura, distribuição de soluto e fração de sólido(porosidade).

$$
R s=\frac{\rho_{l} \varepsilon_{l}}{H_{l}}\left(H_{\text {liquidus }}-H_{T}\right) \frac{v o l}{\Delta t}
$$

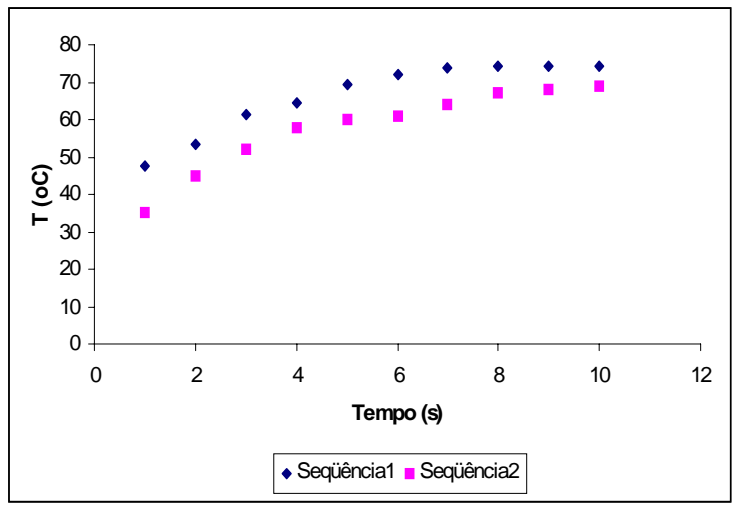

Figura 3b - Valores medidos versus valores previstos, sem a resistência térmica de contato.

Para a condutividade térmica da liga assume-se uma variação linear com a temperatura.

$k=k_{0}(1+\beta T)$

Os valores de $\mathrm{a}, \mathrm{b}, \mathrm{c}, \mathrm{k}_{0}$ e $\beta$ são obtidos nas tabelas de dados termodinâmicos ${ }^{8)}$. 


\section{RESULTADOS}

Os cálculos foram realizados utilizando-se de um software desenvolvido pelos autores ${ }^{2,3)}$, onde estabelecidos as condições iniciais e de contorno o modelo prediz as distribuições de temperatura, fração mássica e fração solidificada. $\mathrm{O}$ domínio para o tem altura $0.012 \mathrm{~m}$, sendo que a região da liga metálica tem uma espessura de $0.002 \mathrm{~m}$. A primeira condição se fez necessário, pois a altura do molde é de $0.012 \mathrm{~m}$, malhas um pouco mais grosseiras não detectariam com uma certa sensibilidade variações na região da liga Al-Si, contudo o refino da malha com o objetivo de detectar variações na região da liga eleva consideravelmente o tempo total de cálculo. A extração de calor através do molde para a atmosfera é extremamente lenta, quando comparado com o aquecimento do molde. $O$ que leva à conclusão que o principal parâmetro que governa a extração de calor a partir da liga metálica são as propriedades de condução do molde e principalmente a resistência térmica de contato.

A figura 2 mostra o campo de temperaturas tridimensional do sistema metal $/$ molde. O perfil de temperatura tridimensional previsto pelo modelo representa o resultado para o tempo de 1 segundo e um coeficiente de convecção de $40 \mathrm{~W} / \mathrm{m}^{20} \mathrm{C}$.

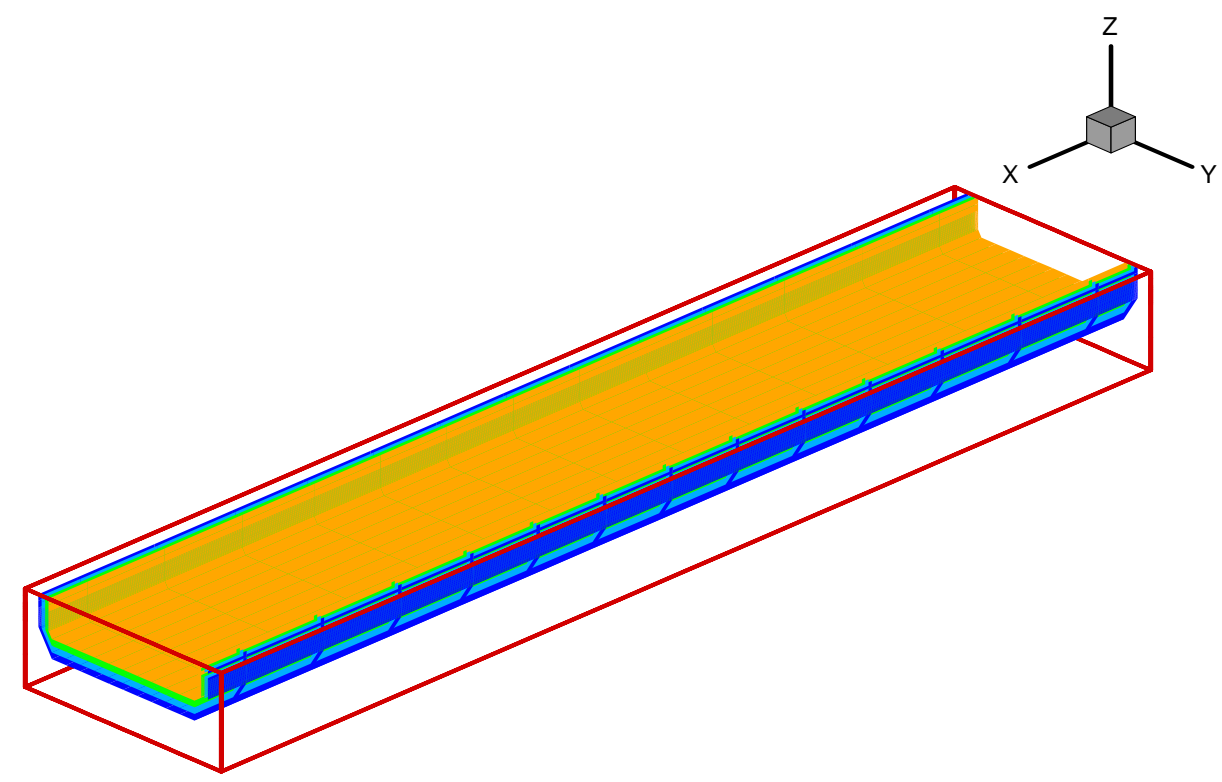

Figura 4 -Fração solidificada da tira para a simulação do lingotamento contínuo de tiras.

Observa-se na figura 2, que a maior temperatura calculada pelo modelo é de $224,8^{\circ} \mathrm{C}$ e a menor de $78,7^{\circ} \mathrm{C}$, para as condições acima citadas. Pode-se notar que as menores temperaturas se encontram na região do molde, isto mostra a influência do molde sobre a solidificação da liga. A temperatura do molde aumenta lentamente enquanto a temperatura da tira decai rapidamente, isto resulta em valores de temperatura próximos à temperatura inicial em grande parte do molde nos instantes

ENGEVISTA, v. 6, n. 1, p. 35-46, abr. 2004 iniciais, onde a liga solidifica rapidamente transferindo a energia devido ao calor latente para o molde.

Nas figuras $3 a$ e $3 b$ são apresentados os resultados para a temperatura de valores calculados pelo modelo e medidos para a região do molde, nesta região foram posicionados termopares ligados a um sistema de aquisição de dados de forma a registrar em intervalos de 1 em 1 segundo as 
temperaturas e compara-las com os valores calculados pelo modelo.

A figura 3 a mostra os valores para as temperaturas medidas e calculadas pelo modelo, levando-se em consideração a resistência térmica de contato entre $o$ molde e a tira. Pode-se observar que os valores previstos pelo modelo (sequência 1) estão próximas daquelas medidas (sequência 2), o que numa etapa inicial serve como validação do modelo proposto. $\mathrm{Na}$ figura $3 \mathrm{~b}$, os valores calculados para a temperatura são comparados com os valores medidos, estes cálculos foram realizados desprezando-se a resistência térmica de contato, observa-se que para estas condições os valores calculados estão acima daqueles medidos para a temperatura, portanto confirmando que a resistência térmica de contato exerce papel crucial no resfriamento da tira.

\subsection{ESTUDO DA INFLUENCIA DA VELOCIDADE DE LINGOTAMENTO SOBRE A TEMPERATURA DA TIRA}

Para estes cálculos foi considerada a tira em movimento, simulando o processo contínuo de lingotamento de tiras. A figura 4 mostra a espessura da casca solidificada após $1 \mathrm{~s}$, tempo necessário para o preenchimento completo do molde.

A figura 5 , mostra a variação da temperatura em virtude da variação da velocidade de lingotamento.

$\mathrm{Na}$ figura 5 , podemos observar que à medida que a velocidade de lingotamento da tira é aumentada há o aumento da temperatura da tira. Isto se deve ao fato que quanto maior a velocidade de lingotamento da tira menor é o tempo de contato metal/molde, conseqüentemente a temperatura da tira num ponto fixo do molde tenderá a aumentar. Estas temperaturas foram calculadas para um coeficiente de convecção de $40 \mathrm{w} / \mathrm{m}^{20} \mathrm{C}$ e, ainda considerando-se a resistência térmica de contato metal $/$ molde.

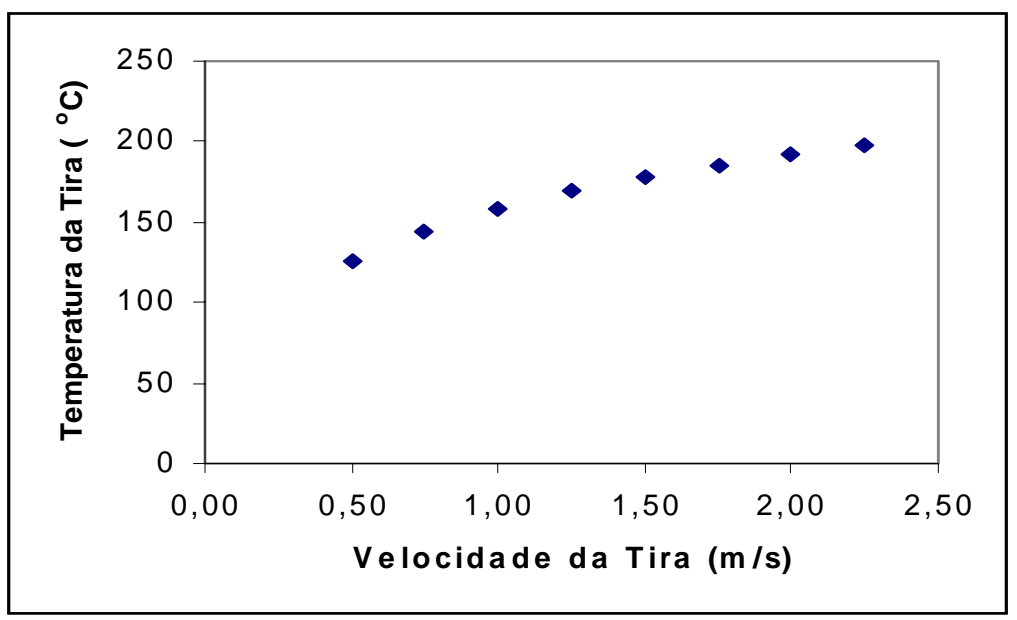

Figura 5 - Temperatura da tira de Al-Si versus a velocidade da lingotamento da tira. 


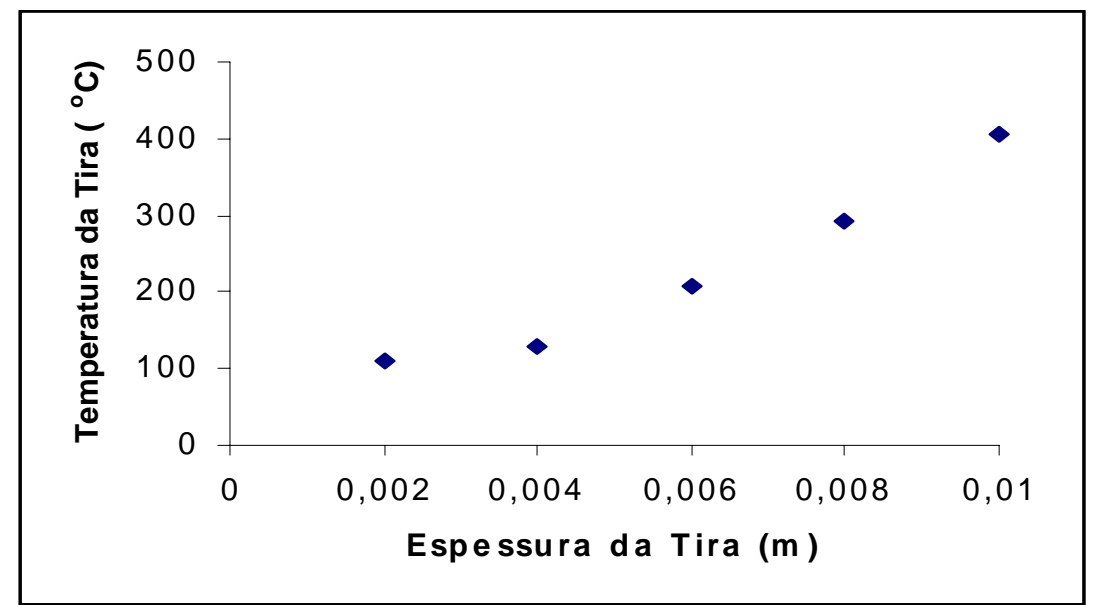

Figura 6 - Espessura da Tira de Al-Si versus a Temperatura.

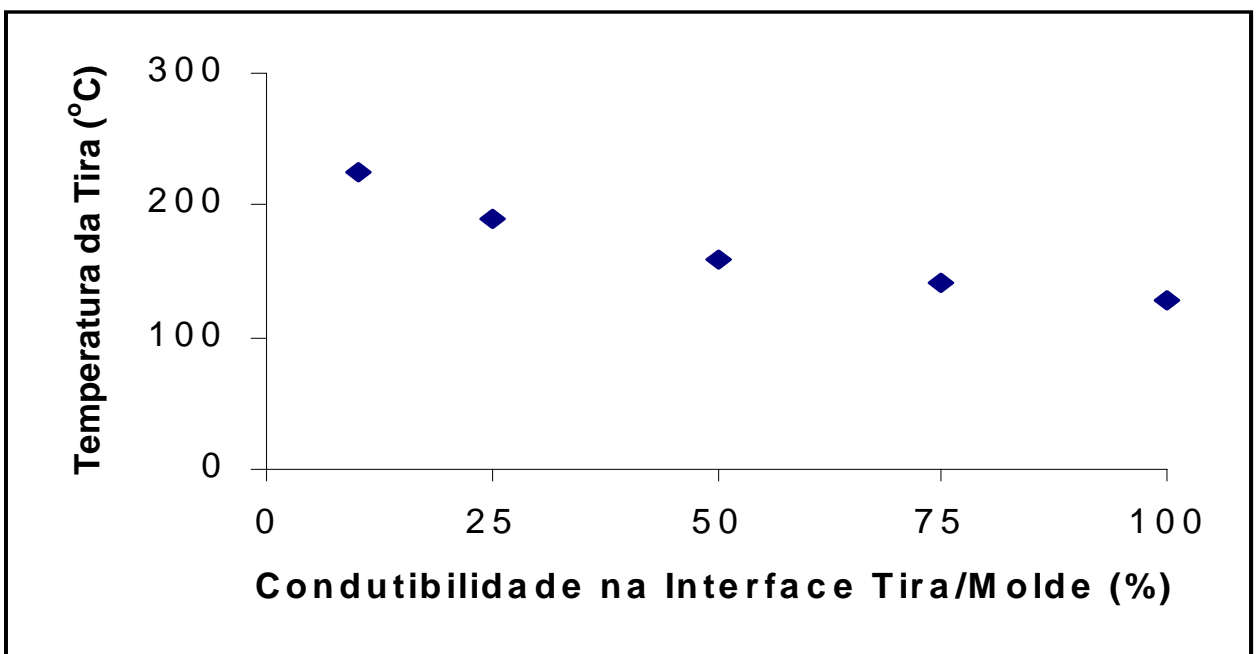

Figura 7 - Condutibilidade de Contato versus Temperatura da Tira.

3.2. ESTUDO DA INFLUENCIA DA ESPESSURA DA TIRA SOBRE A TEMPERATURA

A figura 6 , mostra a espessura da tira plotada contra a temperatura da mesma. Para estes cálculos a velocidade da tira foi de $1 \mathrm{~m} / \mathrm{s}$, e o coeficiente de convecção de $40 \mathrm{~W} / \mathrm{m}^{20} \mathrm{C}$. Nesta figura pode-se observar que a medida que a espessura da tira aumenta, também aumenta a temperatura da mesma, isto se ENGEVISTA, v. 6, n. 1, p. 35-46, abr. 2004 deve ao fato de haver mais material para se resfriar, ao se aumentar a espessura da mesma.

\subsection{ESTUDO DA INFLUÊNCIA DA CONDUTIBILIDADE DE CONTATO SOBRE A TEMPERATURA DA TIRA.}

A figura 7, mostra a resistência térmica de contato tira/molde plotada contra a temperatura da tira, neste cálculo 
foram mantidas constante as dimensões da tira, o coeficiente de convecção e a velocidade de lingotamento.

$\mathrm{Na}$ figura 7, pode-se observar que com o aumento da condutibilidade de contato tira/molde, a temperatura da tira decresce, ou seja, com o aumento da condutibilidade na interface maior calor é transferido para o molde, como consequência tem-se o resfriamento da tira. A estratégia adotada neste trabalho foi aumentar a condutibilidade na região entre a tira e o molde, visando simular condições ótimas de lingotamento.

\section{CONCLUSÕES}

De modo geral, os resultados obtidos neste trabalho confirmam os estudos prévios com relação à importância da resistência térmica de contato. Os resultados da simulação mostram que as propriedades do molde são de fundamental importância, pois este funciona como um reservatório térmico, sendo sua capacidade de difusão de calor um fator limitante da extração de calor da tira e conseqüente taxa de solidificação, confirmando os resultados obtidos por Netto et alli $^{3)}$ e Guthrie e Tavares ${ }^{2)}$.

O aumento na velocidade de lingotamento da tira, promove o aumento da temperatura num determinado ponto da tira, em relação ao tempo, isto se deve ao fato de haver menor tempo de contato entre molde e tira para a transferência de calor.

$O$ aumento da espessura da tira promove o aumento da temperatura num determinado ponto, em relação ao tempo. Isto se deve ao fato, de haver maior volume de material para ser resfriado.

$\mathrm{O}$ aumento da condutibilidade de contato promove a diminuição de temperatura num determinado ponto, em relação ao tempo. Isto se deve ao fato, de haver uma quantidade maior de calor sendo transferida para o molde.

Dentre as formas identificadas para a transferência de calor, especial atenção deve ser dada a transferência de calor

ENGEVISTA, v. 6, n. 1, p. 35-46, abr. 2004
Newtoniana, uma vez que esta controla toda a taxa de extração de calor entre metal e molde, resultado também confirmado por outros pesquisadores ${ }^{3)}$.

Os cálculos realizados admitindo a resistência térmica de contato estão de acordo com os valores obtidos experimentalmente, o que para uma etapa inicial serve como validação do modelo proposto.

Os valores calculados pelo modelo, desprezando-se a resistência térmica de contato, superestimam os valores experimentais.

\section{NOMENCLATURA}

A: Área $\left(\mathrm{m}^{2}\right)$

Cp: Calor específico $\quad\left(\mathrm{kJ} / \mathrm{kg}{ }^{\circ} \mathrm{C}\right)$

$D_{i}^{j}$ : Difusividade binária da espécie i em $\mathrm{j}$

$\left(\mathrm{m}^{2} / \mathrm{s}\right)$

$\mathrm{h}_{\mathrm{i}}$ : Entalpia da fase i $(\mathrm{kJ} / \mathrm{kg})$

$\mathrm{h}_{\mathrm{w}}$ :Coeficiente de convecção global $\left(\mathrm{W} / \mathrm{m}^{2}{ }^{\circ} \mathrm{C}\right)$

$h_{\text {aparente: }}$ Coeficiente de transferência de calor newtoniano $\left(\mathrm{W} / \mathrm{m}^{2}{ }^{\circ} \mathrm{C}\right)$

$\mathrm{H}_{\mathrm{L}}$ : Calor latente de solidificação $(\mathrm{kJ} / \mathrm{kg})$

$\mathrm{k}$ : condutividade térmica $\left(\mathrm{W} / \mathrm{m}{ }^{\circ} \mathrm{C}\right)$

q: calor transferido (W)

R: taxa de solidificação $\left(\mathrm{kmol} / \mathrm{m}^{3} \mathrm{~s}\right)$

$\mathrm{S}$ : termo fonte da equação de transporte (varias unidades, dependendo da equação)

$\mathrm{t}$ : tempo (s)

$\mathrm{T}$ : temperatura $\left({ }^{\circ} \mathrm{C}\right)$

$\mathrm{U}$ : velocidade da fase $(\mathrm{m} / \mathrm{s})$

$\mathrm{x}, \mathrm{y}, \mathrm{z}$ : coordenadas espaciais $(\mathrm{m})$

$\varepsilon$ :fração volumétrica da fase

$\rho:$ densidade da fase $\left(\mathrm{kg} / \mathrm{m}^{3}\right)$

$\varphi$ :emissividade térmica ( )

\section{REFERÊNCIAS}

1. VISKANTA,R., BIANCHI,M.V., CRITSER, J.K. and GAO, D. Solidification processes of solutions, Cryobiology,v34(1997), pp.348-362.

2. GUTHRIE,R.I.L. and TAVARES, R.P., Mathematical and physical 
modeling of steel flow and solidification in twin-roll/horizontal belt thin-strip casting machines, Applied Mathematical modelling, v22(1998), pp851-872.

3. NETTO, P.G.Q, Mathematical and physical modelling of a single belt casting process, Ph.D Thesis, McGill University, Montreal, Canadá, July, (1998)

4. SILVA A. T.: Influência da forma de deposição de metal na qualidade de tiras produzidas em máquina de lingotamento de cinto único Tese de mestrado apresentada ao programa de pós graduação em engenharia metalúrgica UFF/EEIMVR - Volta Redonda - RJ - Brasil (2002).

5. JUNIOR J. M. F.: Construção de um simulador de lingotamento contínuo de tiras: da idéia ao protótipo. Tese de mestrado apresentada ao programa de pós graduação em engenharia metalúrgica UFF/EEIMVR - Volta Redonda - RJ - Brasil (2001).

6. SLATTERY J.C. Momentum, energy, and mass transfer in continua. McGraw-Hill Chemical engineering series, 1972
7. CASTRO J.A, NOGAMI H. YAGI J: Transient mathematical model of the blast furnace based on multi-fluid concept, with application to high PCI operation, ISIJ International, Tokyo, vol 40, n.7, p637-646 Jul.,2000

8. CASTRO J.A, NOGAMI H. YAGI $\mathrm{J}$ :Three-dimensional multiphase mathematical model of the blast furnace based on the multifluid model, ISIJ International , Tokyo, vol 42, n.1 p44-52, Jan. 2002.

9. MELAAEN M.C.:Calculation of fluid flows with staggered and nonstaggered curvilinear nonothogonal grids-the theory, Numerical heat transfer B vol 21 p1-19, 1992

10. PATANKAR S.V: Numerical heat transfer and fluid flow. Hemisphere Publishing Co. Series in computational Methods in mechanical and thermal science, 1980

11. SHACKELFORD J.F: Introduction to materials science for engineers. $3^{\text {rd }}$ edition Macmillan Publishing Co.,1992

12. PERRY R. and GREEN, D. : Chemical Engineering Handbook, $6^{\text {th }}$ edition , USA, MacGraw-Hill, 1984 